\title{
Will Artificial Intelligence Replace Nurses? A Debate
}

\author{
Laura-Maria PELTONEN ${ }^{\mathrm{a}, 1}$, Lisiane PRUINELLI ${ }^{\mathrm{b}}$, Adrienne LEWIS $^{\mathrm{c}}$, \\ Lorraine BLOCK ${ }^{\mathrm{d}}$, Maxim TOPAZ ${ }^{\mathrm{e}}$, Hanna von GERICH $^{\mathrm{a}}$ and \\ Charlene RONQUILLO ${ }^{\mathrm{f}}$ \\ ${ }^{a}$ Department of Nursing Science, University of Turku, Turku, Finland \\ ${ }^{\mathrm{b}}$ School of Nursing, University of Minnesota, Minneapolis, Minnesota, USA \\ ${ }^{\mathrm{c}}$ Nursing Informatics Consultant, University of Victoria, Victoria, Canada \\ ${ }^{\mathrm{d}}$ School of Nursing, University of British Columbia, Vancouver, Canada \\ ${ }^{\mathrm{e}}$ School of Nursing, Columbia University, New York, New York, USA \\ ${ }^{\mathrm{f}}$ School of Nursing, University of British Columbia Okanagan, Kelowna, Canada
}

\begin{abstract}
Technological development has enabled Artificial Intelligence (AI) to better support health care delivery and nursing. The need for nurses to be involved and steer the development and implementation of AI in health care is recognized. A 60 -minute scientific debate is organized to explore if AI will replace nursing.
\end{abstract}

Keywords. Artificial Intelligence, nursing informatics, health technology

\section{Introduction}

Technological development and digitalization has enabled the progress of Artificial Intelligence (AI) to better support health care delivery and nursing. AI can be defined as "systems that show intelligent behaviour: by analysing their environment they can perform various tasks with some degree of autonomy to achieve specific goals" [1]. Arguably, AI technologies have been used in nursing practice for decades, albeit not necessarily recognized as AI. Nevertheless, the growing interest in AI in healthcare is accompanied by new conversations on the relationship between AI and nursing. There is a need for the nursing profession to engage in the discourse and better understand AI. [2]

AI can have unintended effects, e.g. biases in healthcare delivery [3]. Some other current concerns include the "black box" nature of AI systems; the limited ability of nurses to fully understand how decisions made by AI systems are reached. This raises the need for AI systems that are explainable and transparent. [4] The necessity of nurses' involvement in steering the development and implementation of AI technologies in the health care setting is recognized to be of great importance [5].

The numerous considerations and implications of increasing incorporation of AI in nursing - both positive and negative - demand discussion in the nursing informatics community. AI can cause harm to patients, nurses, and the profession overall. Concurrently, there are great potential benefits for AI to bolster the profession, serve as

${ }^{1}$ Corresponding Author, Laura-Maria Peltonen, Department of Nursing Science, 20014 University of Turku, Finland; E-mail: laura-maria.peltonen@utu.fi. 
a powerful tool for nurses, and improve care delivery and population outcomes. The purpose of this panel, which is organized by the IMIA Student and Emerging Professionals Group (SEP), is to hold a debate about the role of AI in nursing.

\section{Debating the Role of AI in Nursing}

This formal debate will discuss the following proposed motion: "Artificial intelligence will replace nurses". The panelists will present arguments based on high-quality research for and against the motion. The content will include, but is not limited to, perspectives on education, competencies, relevance, safety, privacy, security as well as leadership issues related to AI technologies available for nursing. The audience is encouraged to participate in the debate throughout the process. The duration of the debate is 60 minutes. The running order of the debate is presented in table 1 .

Table 1. Running order of debate.

\begin{tabular}{lr}
\hline Item & $\begin{array}{c}\text { Time } \\
\text { (minutes) }\end{array}$ \\
\hline Introduction and opening of the debate (Laura-Maria Peltonen) & 8 \\
First panelist (Lisiane Pruinelli) presents arguments for the proposed motion & 3 \\
First panelist (Max Topaz) presents arguments against the proposed motion & 3 \\
Second panelist (Adrienne Lewis) presents arguments for the motion & 2 \\
Second panelist (Hanna von Gerich) presents arguments against the motion & 2 \\
Audience participation & 10 \\
Third panelist (Lorraine Block) closing statement for the motion & 2 \\
Third panelist (Charlene Ronquillo) closing statement against the motion & 2 \\
Audience participation & 20 \\
Facilitator closes debate (Laura-Maria Peltonen) & 8 \\
\hline
\end{tabular}

\section{The Panel}

Facilitator: Laura-Maria Peltonen, PhD, MNSc, RN, FEANS, is a researcher at the Department of Nursing Science at the University of Turku, Finland. She is the co-chair for the IMIA SEP Group. Her research targets information utilization to support decision-making of professionals in health service delivery. She is a member of the IKITK research consortium that develops clinical information and language technology solutions for health care.

\subsection{Panelists for the Proposed Motion}

Lisiane Pruinelli, $\mathrm{PhD}, \mathrm{MS}, \mathrm{RN}, \mathrm{FAMIA}$ is an Assistant Professor at the School of Nursing and Affiliate Faculty Institute for Health Informatics, University of Minnesota. Her research is on applying cutting-edge informatics tools and Big Data science methodologies to improve health outcomes for complex disease conditions. Dr. Pruinelli's research on large and heterogeneous clinical data, takes a multidisciplinary approach to estimate effects of evidence-based interventions on health outcomes.

Adrienne Lewis, RN, BsN, MNSc, MS, received her Masters of Science in Health Informatics and Masters of Science in Nursing, in 2017; the first dual faculty program of 
its kind in North America, at the University of Victoria, British Columbia, Canada. Her thesis interest was culturally safe nursing practices and whether they could be defined, and if so, are they represented in standardized nursing languages. Following the completion of her study concerning cultural safety, she continues to design projects that enable nurses working in Indigenous communities access to regional health information systems. She is secretary for the IMIA SEP group.

Lorraine Block is a Registered Nurse with clinical and emerging research experience in the field of nursing informatics. She is currently in her fourth year of $\mathrm{PhD}$ studies at the University of British Columbia, School of Nursing. Her dissertation is related to the computerized representation of nursing wound care knowledge. Lori also works as a clinical leader in Vancouver, British Columbia, where she advances the development, implementation, innovation and analysis of nursing electronic documentation. She is the current chair of the Nursing Informatics Working Group within the IMIA SEP group.

\subsection{Panelists Against the Proposed Motion}

Maxim Topaz is an Elizabeth Standish Gill Associate Professor of Nursing at Columbia University School of Nursing, Columbia Data Science Institute and the Visiting Nurse Service of New York. He earned his PhD degree as a Fulbright Fellow at the University of Pennsylvania and his Master's and Bachelor's degrees from the University of Haifa, Israel. He completed a postdoctoral fellowship at the Harvard Medical School and Brigham Women's Hospital. He published more than sixty articles on topics related to health informatics and his current work focusses on identifying patients at risk for poor outcomes using cutting-edge data science methods (e.g. NLP and machine learning). He is co-chair of the IMIA SEP group.

Hanna von Gerich is a Registered Nurse and Research Assistant at the Department of Nursing Science at the University of Turku in Finland. Her research explores the application of AI technologies in nursing. She is the incoming chair of the Nursing Informatics Working Group within the IMIA SEP group.

Charlene Ronquillo is an Assistant Professor at the School of Nursing, University of British Columbia Okanagan in Canada. Her research is in nursing and health informatics, implementation science and the intersection of these fields. Prior to her current role, Charlene was an Associate Research Fellow in implementation science at the University of Exeter in the United Kingdom. She is the current vice-chair of the IMIA SEP group.

\section{References}

[1] European Commission 2019. Factsheet: Artificial Intelligence for Europe. Available at: https://ec.europa.eu/digital-single-market/en/news/factsheet-artificial-intelligence-europe

[2] Erikson H, Salzmann-Erikson M. Future challenges of robotics and artificial intelligence in nursing: what can we learn from monsters in popular culture? The Permanente Journal, 20(3), (2016)

[3] Parikh RB, Teeple S1, Navathe AS1,2. Addressing bias in artificial intelligence in health cCare. JAMA. 2019 Nov 22. doi: 10.1001/jama.2019.18058. [Epub ahead of print]

[4] Holzinger A, Biemann C, Pattichis CS, Kell DB. What do we need to build explainable AI systems for the medical domain? ( 2017) arXiv preprint arXiv:1712.09923.

[5] Glauser W. Artificial intelligence, automation and the future of nursing, The Canadian nurse 3 (2017), 24-26. 